\section{Transmissão vertical do HIV, da sífilis e da hepatite B no município de maior incidência de AIDS no Brasil: um estudo populacional no período de 2002 a 2007}

\section{Vertical transmission of HIV, syphilis and hepatitis $B$ in the municipality with the highest incidence of AIDS in Brazil: a population-based study from 2002 to 2007}

\section{Emil Kupek'}

Juliana Fernandes de Oliveira"

' Departamento de Saúde Pública da Universidade Federal de Santa Catarina/ UFSC.

" Universidade do Vale do Itajaí /UNIVALI

\section{Resumo}

Objetivo: O objetivo deste trabalho foi verificar a prevalência em gestantes e a taxa de transmissão vertical do HIV, sífilis e hepatite B no município de Itajaí - SC, no período de 2002 a 2007. Métodos: Este foi um estudo longitudinal retrospectivo, de base populacional, compreendendo os períodos de 2002 a 2007 para HIV e 2004 a 2007 para sífilis e hepatite B, utilizando os dados sistematizados dos sistemas de informação estadual e municipal. Resultados: A prevalência das infecções virais em gestantes foi de $1,7 \%$ para HIV, 0,41\% para hepatite B aguda, $0,46 \%$ para hepatite $B$ crônica e $0,43 \%$ para sífilis. A transmissão vertical do HIV foi de $6,28 \%$, sendo menor que $5 \%$ quando HIV foi diagnosticado antes ou durante a gravidez, comparado com $20 \%$ e $55 \%$ quando o diagnóstico foi feito durante ou após o parto. A transmissão vertical da sífilis foi de $68,89 \%$. Não houve uma tendência estatisticamente significativa da transmissão vertical segundo ano de diagnóstico ou faixa etária para HIV e sífilis. Quase $44 \%$ das gestantes infectadas por HIV conheciam seu status sorológico antes da gravidez; a transmissão vertical neste grupo ficou menor que $5 \%$. Não foi registrado nenhum caso da transmissão vertical da hepatite B. Conclusões: A transmissão vertical do HIV atingiu a meta do Ministério da Saúde quando o diagnóstico foi feito durante o pré-natal, mas foi expressivamente elevada quando o diagnóstico ocorreu somente no parto. A transmissão vertical da sífilis foi muito maior do que a preconizada, mostrando a importância de se reforçar os procedimentos estabelecidos para diminuí-la.

Palavras-chaves: HIV. Sífilis. Hepatite B. Transmissão vertical de doença infecciosa. Efetividade. Saúde da mulher. 


\section{Abstract}

Objective: The objective of this study was to verify the prevalence and vertical transmission rate of HIV, syphilis and hepatitis B in pregnant women in the municipality of Itajaí, state of Santa Catarina, Brazil, during the 2002-2007 period. Methods: Data were collected in a retrospective population-based longitudinal study using computerized medical records of the state and local health authorities during the 2002-2007 period for HIV, and 2004-2007 for syphilis and hepatitis B. Results: The prevalence of HIV, acute hepatitis B, chronic hepatitis B and syphilis in pregnant women was $1.7 \%, 0.41 \%, 0.46 \%$, and $0.43 \%$, respectively. Overall, vertical transmission of HIV was $6.28 \%$, although it was less than $5 \%$ among women diagnosed with HIV before or during pregnancy, compared to $20 \%$ and $55 \%$ among women first diagnosed with HIV during and after delivery, respectively. Vertical transmission of syphilis was $68.89 \%$. No trend was confirmed for the transmission rate either regarding the year of diagnosis or age group of pregnant women. Almost $44 \%$ of HIV infected pregnant women knew their HIV status before becoming pregnant; the HIV transmission rate for these women was less than $5 \%$. No case of vertical transmission was observed for hepatitis B. Conclusions: The vertical transmission rate for HIV was within the target of the Ministry of Health when HIV diagnosis was made during pregnancy, but increased sharply when the diagnosis was made only at delivery. Vertical transmission of syphilis was much higher than expected, showing the importance of reinforcing the procedures recommended for its reduction.

Keywords: HIV. Syphilis. Hepatitis B. Vertical transmission of infectious diseases. Effectiveness. Women's health.

\section{Introdução}

A gravidez é um período de risco aumentado para aquisição de doenças sexualmente transmissíveis (DST), devido à ocorrência de modificações no sistema imunológico que predispõem a gestante a doenças infecciosas, tornando-se um dos problemas mais comuns do período gestacional $^{1}$. Entre suas consequências estão a infertilidade e a transmissão da mãe para o filho, determinando perdas fetais, malformações congênitas e até mesmo a morte, caso não tratadas, além de aumentar os riscos de infecção pelo $\mathrm{HIV}^{2,3}$. Apesar disso, as políticas públicas voltadas para as DST e prevenção de sua transmissão vertical só voltaram a readquirir importância como problema de saúde pública após a epidemia de AIDS, pois estudos mostraram que pessoas com DST podem ter um aumento em até 18 vezes no risco de infecção pelo HIV, além de transmitir mais facilmente o HIV para seus parceiros sexuais ${ }^{4-6}$.

O HIV/AIDS é uma doença sexualmente transmissível que também possui como meio de transmissão as vias parenteral e vertical. Atualmente, observa-se o aumento proporcional de casos de AIDS de transmissão heterossexual e taxas de incidência crescentes no gênero feminino. Este fenômeno, chamado "feminização", é acompanhado por um número cada vez maior de crianças atingidas ${ }^{7,8}$.

A transmissão vertical do HIV pode ocorrer durante a gestação (35\%), o trabalho de parto e o parto propriamente dito (65\%), ou através da amamentação, com risco acrescido de transmissão entre $7 \%$ e $22 \%$ a cada exposição (mamada) ${ }^{9}$. Sem intervenções de profilaxia, ocorre em cerca de $26 \%$ das gestações, podendo ser reduzida para menos de $2 \%$ com intervenções preconizadas pelo Programa Nacional de DST/AIDS, tais como o uso de antirretrovirais combinados a partir da $14^{\text {a }}$ semana de gestação, utilização de AZT injetável durante o trabalho de parto e o parto, parto por cirurgia cesariana eletiva, AZT oral para o recém-nascido exposto do nascimento 
até 42 dias de vida e inibição da lactação associada ao fornecimento de fórmula infantil até os seis meses de idade ${ }^{8,9}$.

A transmissão vertical da sífilis pode alcançar taxas entre $70 \%$ e $100 \%$ em gestantes não tratadas, através das espiroquetas que atingem a placenta e causam endarterite (aumento da resistência das artérias uterinas e umbilicais) e, consequentemente, abortos tardios, prematuridade, óbito fetal e sífilis neonatal congênita ${ }^{8,10}$. No entanto, mesmo com instrumentos diagnósticos (teste VDRL) e terapêuticos (penicilina benzatina) eficazes, baratos e de execução simples, a doença continua sendo um problema de saúde pública mundial, inclusive no Brasil ${ }^{11-13}$. O mesmo paradoxo se estende para a hepatite $B$, onde a transmissão vertical pode alcançar $95 \%$ quando há replicação viral, mas este risco pode ser reduzido em $90 \%$ com imunoglobulina e vacina contra hepatite B, aplicadas até as primeiras 12 horas de vida do recém-nascido ${ }^{10}$. Entre $70 \%$ e $90 \%$ dos recém-nascidos infectados desenvolvem a forma crônica da doença e 20-25\% destes evoluem para doenças hepáticas avançadas, principalmente a cirrose $\mathrm{e}$ o hepatocarcinoma ${ }^{14}$.

Apesar da reconhecida importância da transmissão vertical para a saúde pública, ainda existem poucas publicações sobre o assunto nas revistas científicas brasileiras, principalmente para sífilis e hepatite B. Com relação ao HIV, destaca-se por sua abrangência um estudo realizado em 17 maternidades públicas de referência em 4 capitais brasileiras, que mostrou a taxa de transmissão vertical de $5,58 \%$ no período de 1997 a $2003^{13}$. Outros estudos de menor abrangência mostraram uma grande variação nas taxas de transmissão vertical do HIV: houve uma redução de $32,3 \%$ para $2,9 \%$ em Campinas - SP na década de $1990^{14}$; em Porto Alegre - RS as taxas variaram entre $2,8 \%$ e $8,31 \%$ na década de $2000^{15,16}$, semelhante aos $5,95 \%$ encontrados em Novo Hamburgo - RS ${ }^{17}$ e 5,1\% em Juiz de Fora - $\mathrm{MG}^{18}$, mas inferiores aos $9,7 \%$ encontrados em Santos - SP ${ }^{19}$. A taxa de 2,4\% encontrada em Rio de Janeiro no período de
1996 a $2004^{20}$ foi muito próxima a de 2,5\% em Campo Grande - MS ${ }^{21}$.

No Brasil, a transmissão vertical da sífilis é monitorada indiretamente, pela incidência de sífilis congênita, a qual usa como denominador o número de nascidos vivos, e não diretamente, dividindo-se o número de filhos infectados pelo número de gestantes com sífilis. A medida direta é muito mais precisa que a indireta, mas exige boa cobertura dos testes para sífilis em gestantes e parturientes, o que continua sendo um ponto fraco do atendimento pré-natal no país. Por exemplo, uma amostra representativa de gestantes no Brasil, nos anos de 1999 e 2000, revelou que 56\% delas não haviam sido testadas para HIV e 41,2\% não haviam sido testadas para sífilis ${ }^{22}$. Nas áreas de baixa renda das regiões Norte e Nordeste do Brasil, os testes de HIV e sífilis em gestantes duplicaram no período de 2002 a 2005, mas ainda permaneceram em um patamar muito baixo de $16 \%^{23,24}$. Além disso, a vigilância epidemiológica raramente faz busca ativa para sífilis congênita, levando a uma grande subnotificação. Em um dos poucos estudos sobre este assunto, a subnotificação foi de $64 \%$ em Palmas, Tocantins ${ }^{25}$.

A pesquisa sobre transmissão vertical da hepatite B é muito rara no Brasil, já que na rede pública de saúde este teste não fazia parte da rotina de pré-natal até o ano de 2005, quando o HBsAg (antígeno de superfície) passou a ser recomendado pelo Ministério da Saúde como marcador de infecção durante a gravidez.

O objetivo deste trabalho é descrever a prevalência em gestantes e a taxa de transmissão vertical de HIV, sífilis e hepatite B no município de Itajaí-SC, no período de 2002 a 2007. O município apresentou a maior incidência de AIDS $(98,1$ por 100.000 habitantes) para o período de 2002-2007 e tem implementado vários projetos para reduzi-la, tais como aprimorar os testes para HIV e VDRL durante gestação e acrescentar como obrigatório o teste para hepatite B (visto que essa doença também é transmitida sexualmente), assim como oferecer o tratamento gratuito para estas doenças. 


\section{Métodos}

O estudo foi delineado como longitudinal e retrospectivo, de base populacional, compreendendo os períodos de 2002 a 2007 para HIV e 2004 a 2007 para sífilis e hepatite $\mathrm{B}$, conforme a disponibilidade de dados sistematizados nos sistemas de informação SINASC, SIM e SIH do Estado de Santa Catarina, e SINAN para o município de Itajaí - SC. As fontes de dados foram utilizadas da seguinte forma: SINASC para as gestantes com nascidos vivos; SIM para as gestantes que tiveram natimortos ou quando ocorreu a morte materna antes do parto; SIH para informações sobre abortamentos; SINAN para notificações e investigações de gestante com HIV e criança exposta, sífilis em gestante, sífilis congênita e hepatites virais; os registros do Laboratório Municipal de Itajaí para marcadores sorológicos da hepatite B e as solicitações de imunoglobulina anti-hepatite B para os recém-nascidos expostos; e as informações fornecidas pelo Programa Municipal de Hepatites Virais sobre os recém-nascidos expostos à hepatite B. As duas fontes citadas por último foram necessárias porque a hepatite $\mathrm{B}$ em gestante não foi de notificação compulsória e a ficha de investigação de hepatites virais só passou a contemplar o campo "gestante" a partir de 2007.

As informações necessárias foram unificadas num arquivo usando o nome da mãe como campo chave. A apuração dos dados envolveu principalmente as formas semelhantes, porém não idênticas, do nome da mãe, verificando outros dados pessoais para confirmar se os nomes diferentes pertenciam a pessoas distintas ou não.

Entre 15.098 gestantes no período de 2002 a 2007 (Tabela 1), 311 (2,06\%) não foram incluídas na análise de transmissão vertical, sendo que 197 destas tiveram aborto, 104 tiveram natimortos, 6 morreram antes do parto ou aborto e 4 não deram informações sobre desfecho gestacional. Os casos de natimortos (óbitos fetais) foram extraídos do SIM através do nome da mãe. A definição do natimorto no SIM é a morte do produto da gestação antes da expulsão ou de sua extração completa do corpo materno, independentemente da duração da gravidez. Indica o óbito ou fato de, depois da separação, o feto não respirar nem dar nenhum outro sinal de vida, como batimentos do coração, pulsações do cordão umbilical ou movimentos efetivos dos músculos de contração voluntária ${ }^{26}$.

Entre 14.787 gestantes com informações suficientes para análise da transmissão vertical, 151 tiveram gestações múltiplas, sendo 149 duplas, uma tripla e uma quádrupla, resultando em 14.941 nascidos vivos. Das 251 gestantes soropositivas pelo HIV e acompanhadas no período de estudo, oito tiveram aborto e duas morreram antes do parto, resultando em 243 recém-nascidos expostos a transmissão vertical (duas gestações eram gemelares). Para o cálculo da taxa de transmissão vertical, 36 destes foram excluídos, sendo 2 casos de óbito fetal por causas não relacionadas ao HIV e 34 perdas de seguimento do recém-nascido por mudança do endereço da mãe. Entre as 44 gestantes diagnosticadas com sífilis durante a gravidez, ocorreram dois abortos e dois natimortos por sífilis congênita, que foram incluídos no cálculo da taxa de transmissão vertical, acrescentando ainda um caso de sífilis congênita diagnosticado somente após o parto. No período de 2004 a 2007, em que foi implementado o monitoramento para sífilis e hepatite B, foram registradas 10.147 gestantes que geraram 9.922 nascidos vivos, sendo que ainda ocorreram 152 abortos, 65 natimortos, 4 mortes maternas e 4 perdas de acompanhamento por mudança de endereço da mãe.

Entre as 47 gestantes infectadas pela hepatite B, uma morreu antes do parto e quatro se perderam no acompanhamento por mudança de endereço. Em resumo, as perdas durante a gravidez sugerem uma associação entre as infecções maternas por HIV, sífilis e hepatite B, com desfechos gestacionais adversos. A cobertura da população alvo foi boa para fins do estudo e as perdas provavelmente não comprometem as principais conclusões obtidas. 
O município de Itajaí tem como rotina de pré-natal a realização de sorologia para o HIV, sífilis e hepatite B no $1^{\circ}$ e $3^{\circ}$ trimestres de gestação centralizados no laboratório municipal de referência. A única maternidade do município tem como rotina a realização do teste rápido anti-HIV nas gestantes que não o realizaram durante o pré-natal desde 2002, e a realização do teste VDRL (Venereal Disease Research Laboratory) para todas as gestantes que se internam para parto ou curetagem desde 2005, com cobertura acima de $99 \%$.

As definições de caso e de transmissão vertical utilizadas seguiram as recomendadas da vigilância epidemiológica do Ministério da Saúde ${ }^{6}$. Para fins deste trabalho, consideramos hepatite B aguda confirmada na presença de qualquer um dos seguintes marcadores sorológicos positivos: HBsAg (antígeno de superfície), anti-HBc IgM (anticorpo "core", imunoglobulina classe M) e HBeAg (antígeno "e"). A forma crônica da doença foi definida pela presença do anti-HBc total (anticorpo "core" somando imunoglobulina de classes M e G) positivo e ausência dos demais marcadores citados.

A análise estatística relacionou a transmissão vertical das doenças citadas com fatores sociodemográficos (faixa etária e escolaridade), de saúde reprodutiva (número de filhos nascidos vivos e mortos, número de consultas pré-natal) e o ano de diagnóstico. A transmissão vertical do HIV também foi relacionada ao momento do diagnóstico. A diferença em transmissão vertical da sífilis foi analisada segundo o tipo de tratamento (adequado ou não). O risco relativo (RR) e seu intervalo de confiança (IC) de 95\% nas comparações acima foram determinados via regressão de Poisson com software Stata, utilizando o estimador robusto da variância ${ }^{27}$.

A pesquisa foi aprovada pelo Comitê de Ética em Pesquisa da Universidade Federal de Santa Catarina (processo no. 269/08). Os autores não têm nenhum conflito de interesse.

\section{Resultados}

No período de 2002 a 2007, foram analisadas 15.098 gestantes, sendo 10.147 destas no período de 2004 a 2007, quando foi implementado o monitoramento para sífilis e hepatite B. Os perfis das gestantes com e sem infecção mostraram-se diferenciados com relação a fatores de risco analisados (Tabela 1). Entre as gestantes sadias, a percentagem daquelas com até 20 anos era maior do que entre as infectadas com HIV, sífilis ou hepatite B. As gestantes infectadas pelo HIV ou sífilis tinham menor escolaridade, maior número de gestações prévias e de natimortos, mas menos consultas pré-natais se comparadas com as gestantes sem infecção.

Durante a gravidez, todas as gestantes fizeram os testes para HIV e hepatite B, e no mínimo um teste para sífilis. A prevalência das infecções virais foi de 1,7\% (251/14.787 gestantes) para HIV, 0,41\% (42/10.147 gestantes) para hepatite B aguda e 0,46\% (47/10.147 gestantes) para hepatite B na vida. A prevalência da sífilis em gestantes foi de $0,43 \%$ (44/10147 gestantes).

A transmissão vertical do HIV foi de $6,28 \%$ no total, sendo menor que $5 \%$ quando HIV foi diagnosticado antes ou durante a gravidez, comparado com $20 \%$ e $55 \%$ quando o diagnóstico foi feito durante ou após parto, respectivamente (Tabela 2). Não houve variação estatisticamente significativa na transmissão vertical segundo ano de diagnóstico, faixa etária e AIDS materna.

A transmissão vertical da sífilis foi de $68,89 \%$ no período de 2004 a 2007 (Tabela 3). Não houve uma tendência estatisticamente significativa na transmissão vertical segundo ano de diagnóstico ou faixa etária. Menos da metade das gestantes com sífilis recebeu o tratamento preconizado; mesmo assim, a transmissão vertical ocorreu em um terço delas (Tabela 3). Por outro lado, todas as gestantes que não receberam o tratamento adequado transmitiram sífilis para seus filhos. A transmissão vertical foi de $12,5 \%$ entre 16 gestantes com sífilis, cujos parceiros foram tratados, comparada com 
Tabela 1 - Características sociodemográficas e fatores de risco para todas gestantes $(\mathrm{N}=15.098)$, comparando as sadias e as infectadas com HIV, síflis e hepatite B em Itajaí, 2002-2007.

Table 1 - Socio-demographic characteristics and risk factors for all pregnant women $(N=15,098)$, comparing healthy women to those infected by HIV, syphilis and hepatitis B in Itajaí, 2002-2007.

\begin{tabular}{|c|c|c|c|c|c|}
\hline \multirow{2}{*}{ Fator de risco } & \multirow{2}{*}{$\mathrm{N}$} & \multicolumn{4}{|c|}{ Número (percentagem) das mulheres com fator de risco } \\
\hline & & Sadia $(n=14756)$ & $H I V(n=251)$ & Sífilis ( $n=44)$ & Hepatite $B(n=47)$ \\
\hline \multicolumn{6}{|l|}{ Faixa etária } \\
\hline$<15$ & 84 & $83(0,56)$ & $0(0,00)$ & $1(2,27)$ & $0(0,00)$ \\
\hline $15-20$ & 3759 & $3700(25,07)$ & $43(17,13)$ & $6(13,64)$ & $10(21,28)$ \\
\hline $21-30$ & 7734 & $7550(51,17)$ & $138(54,98)$ & $22(50,00)$ & $24(51,06)$ \\
\hline $31-40$ & 3061 & $2973(20,15)$ & $63(25,10)$ & $13(29,55)$ & $12(25,53)$ \\
\hline$>40$ & 213 & $203(1,38)$ & $7(2,79)$ & $2(4,55)$ & $1(2,13)$ \\
\hline Sem informação & 247 & $247(1,67)$ & $0(0,00)$ & $0(0,00)$ & $0(0,00)$ \\
\hline \multicolumn{6}{|l|}{ Escolaridade } \\
\hline Nenhuma & 93 & $78(0,53)$ & $14(5,58)$ & $1(2,27)$ & $0(0,00)$ \\
\hline $1-3$ anos & 707 & $670(4,54)$ & $34(13,55)$ & $3(6,82)$ & $0(0,00)$ \\
\hline 4-7 anos & 4456 & $4316(29,25)$ & $100(39,84)$ & $24(54,55)$ & $16(34,04)$ \\
\hline 8-11 anos & 7166 & $7047(47,76)$ & $84(33,47)$ & $15(34,09)$ & $20(42,55)$ \\
\hline $12+$ anos & 2393 & $2368(16,05)$ & $18(7,17)$ & $1(2,27)$ & $6(12,77)$ \\
\hline Sem informação & 283 & $277(1,88)$ & $1(0,40)$ & $0(0,00)$ & $5(10,64)$ \\
\hline \multicolumn{6}{|l|}{ Nascidos vivos } \\
\hline Nenhum & 6575 & $6575(44,56)$ & $51(20,32)$ & $8(18,18)$ & $17(36,17$ \\
\hline $1-3$ & 7143 & $7143(48,41)$ & $172(68,53)$ & $28(63,64)$ & $22(46,81)$ \\
\hline $4+$ & 747 & $747(5,06)$ & $27(10,76)$ & $6(13,64)$ & $2(4,26)$ \\
\hline Sem informação & 291 & $291(1,97)$ & $1(0,40)$ & $2(4,55)$ & $6(12,77)$ \\
\hline \multicolumn{6}{|l|}{ Natimortos } \\
\hline Nenhum & 13354 & $13070(88,57)$ & $210(83,67)$ & $33(75,00)$ & $41(87,23)$ \\
\hline 1 ou mais & 1458 & $1408(9,54)$ & $40(15,94)$ & $10(22,73)$ & $0(0,00)$ \\
\hline Sem informação & 286 & $278(1,88)$ & $1(0,40)$ & $1(2,27)$ & $6(12,77)$ \\
\hline \multicolumn{6}{|l|}{ Consultas pré-natal } \\
\hline Nenhuma & 152 & $129(0,87)$ & $19(7,57)$ & $4(9,09)$ & $0(0,00)$ \\
\hline $1-3$ & 736 & $710(4,81)$ & $19(7,57)$ & $6(13,64)$ & $1(2,13)$ \\
\hline $4-6$ & 3910 & $3811(25,83)$ & $73(29,08)$ & $11(25,00)$ & $15(31,91)$ \\
\hline 7 ou mais & 9977 & $9792(66,36)$ & $138(54,98)$ & $22(50,00)$ & $25(53,19)$ \\
\hline Sem informação & 323 & $314(2,13)$ & $2(0,80)$ & $1(2,27)$ & $6(12,77)$ \\
\hline
\end{tabular}

Observação:"N"e" $n$ " representam os tamanhos dos grupos segundo os fatores de risco e os desfechos analisados, respectivamente.

Note: " $N$ " and " $n$ " are the group sizes according to risk factors and outcomes analyzed, respectively

$100 \%$ entre 29 gestantes cujos parceiros não foram tratados, mostrando um significativo aumento de risco relativo de 8,00 (IC 95\% de 1,91 a 33,53). Mais de $93 \%$ das gestantes com sífilis foram diagnosticadas com forma latente da doença.

A incidência de sífilis congênita foi de 3,12 por mil nascidos vivos (31 casos entre 9.922 nascidos vivos no período de 2004 a 2007).
Hepatite B foi observada na população de 10.139 gestantes no período de 2004 a 2007. Houve uma morte e 4 perdas de seguimento entre 47 gestantes portadoras de hepatite B. Nasceram 42 crianças expostas à transmissão vertical da hepatite $\mathrm{B}$, mas apenas 5 foram acompanhadas até o encerramento, nenhuma delas tendo sido infectada. 
Tabela 2 - Transmissão vertical (TV) do HIV em nascidos vivos expostos: risco relativo (RR) e intervalo de confiança (IC) de $95 \%$ segundo o momento de diagnóstico do HIV em gestantes. Itajaí, 2002-2007.

Table 2 - Vertical transmission (TV) of HIV in newborns from HIV-infected mothers: relative risk (RR) and 95\% confidence interval (IC) according to time of HIV diagnosis. Itajaí, 2002-2007.

\begin{tabular}{lcccccc}
\hline Diagnóstico do HIV & $\begin{array}{c}\text { Nascidos vivos } \\
\text { expostos }^{\mathrm{a}}\end{array}$ & $\begin{array}{c}\text { Nascidos vivos } \\
\text { HIV+ }\end{array}$ & TV (\%) & RR & IC 95\% & $\mathrm{Pc}^{\mathrm{c}}$ \\
\hline Antes do pré-natal & 90 & 4 & 4,44 & $1,00^{\mathrm{b}}$ & & \\
Durante o pré-natal & 102 & 2 & 1,96 & 0,44 & 0,$08 ; 2,41$ & 0,345 \\
Durante o parto & 5 & 1 & 20,00 & 4,50 & 0,$50 ; 40,26$ & 0,179 \\
Após o parto & 9 & 5 & 55,56 & 12,50 & 3,$36 ; 46,55$ & $<0,001$ \\
Sem informação & 1 & 1 & 100,00 & 22,50 & 2,$51 ; 201,30$ & 0,005 \\
\hline Total & 207 & 13 & 6,28 & - & - & - \\
\hline
\end{tabular}

a Exclui 4 gestantes com teste HIV positivo sem data, 2 óbitos fetais e 30 perdas de seguimento do recém nascido por mudança do endereço da mãe

a Excludes 4 pregnant women with a positive HIV test result without a date, 2 cases of fetal death not related to HIV and 30 newborn children lost to follow-up due to mothers'change of address

${ }^{\mathrm{b}}$ categoria de referência / ${ }^{\mathrm{b}}$ baseline category

c probabilidade de RR ser igual a de categoria de referência/ ' likelihood that RR equals the baseline category

Tabela 3 - Efeito da adequação do tratamento medicamentoso das gestantes para prevenção da transmissão vertical (TV) da sífilis em nascidos vivos expostos: risco relativo (RR) e intervalo de confiança (IC) de 95\%. Itajaí, 2004-2007.

Table 3 -Effect of the adequacy of pharmacological treatment of pregnant women on preventing vertical transmission (TV) of syphilis in newborns of syphilis-infected mothers: relative risk (RR) and 95\% confidence interval (IC). Itajaí, 2004-2007.

\begin{tabular}{|c|c|c|c|c|c|c|}
\hline \multirow{2}{*}{$\begin{array}{l}\text { Tratamento da } \\
\text { gestante }\end{array}$} & \multirow[t]{2}{*}{ Expostos a TV ${ }^{a}$} & \multicolumn{2}{|c|}{ TV } & \multirow[t]{2}{*}{$\mathrm{RR}$} & \multirow[t]{2}{*}{ IC 95\% } & \multirow[t]{2}{*}{$\mathrm{P}^{\mathrm{c}}$} \\
\hline & & $\mathrm{n}$ & $\%$ & & & \\
\hline Adequado & 21 & 7 & 33,33 & $1,00^{\mathrm{b}}$ & & \\
\hline Inadequado & 3 & 3 & 100,0 & 3,00 & 0,$78 ; 11,60$ & 0,111 \\
\hline Não realizado & 21 & 21 & 100,0 & 3,00 & 1,$27 ; 7,06$ & 0,012 \\
\hline Total & 45 & 31 & 68,89 & - & - & - \\
\hline
\end{tabular}

\section{Discussão}

Antes de comentar os resultados do estudo, convém considerar algumas limitações. A falta da data para o teste VDRL para maioria das gestantes impediu análise de impacto do momento de diagnóstico de sífilis na TV. A falta de acompanhamento por tempo suficiente para confirmar a TV da hepatite B na maioria dos recém-nascidos expostos resultou em somente cinco deles com diagnóstico definido conforme preconizado pelo Ministério da Saúde, impossibilitando uma análise mais aprofundada com amostra tão pequena. A avaliação da adequação do tratamento da sífilis foi restrita ao tratamento medicamentoso das gestantes, porque a maioria das gestantes infectadas não tinha informação sobre o tratamento dos seus parceiros. Finalmente, o período analisado foi bastante curto para estabelecer a significância estatística da tendência temporal com maior precisão, particularmente com relação à sífilis e à hepatite B.

Apesar das limitações, o estudo usou uma base populacional com boa cobertura dos testes sorológicos para HIV, sífilis e hepatite B em gestantes, juntando os dados relevantes de todos os sistemas informatizados de tratamento desta população e verificando-os com busca manual quando 
necessário. Pela revisão da literatura brasileira, este é um dos primeiros estudos de base populacional em um dos municípios do país com mais alta incidência de AIDS.

A transmissão vertical do HIV de 6,28\% no município de Itajaí encontra-se acima da meta do Ministério da Saúde, de 2\%, alcançável através de todas as intervenções profiláticas preconizadas. Percebe-se que a meta foi alcançada quando o diagnóstico do HIV foi feito durante o pré-natal, mas o risco da transmissão ficou 4,5 vezes maior quando o diagnóstico ocorreu no parto e 12,5 vezes maior quando HIV foi diagnosticado após o parto, reforçando a pertinência dos procedimentos preconizados pelo Ministério da Saúde. É importante notar que quase $44 \%$ das gestantes infectadas por HIV conheciam seu status sorológico antes da gravidez e que a transmissão vertical neste grupo ficou menor que $5 \%$.

A taxa geral de transmissão vertical do HIV em Itajaí foi menor que a taxa de $8,4 \%$ estimada para o Brasil no ano de 2004 e dentro da faixa de resultados encontrados na maioria dos estudos brasileiros, tais como 6,62\% em Porto Alegre - RS ${ }^{16}, 5,95 \%$ em Novo Hamburgo - $\mathrm{RS}^{17}$ e 5,58\% em estudo multicêntrico realizado nas 4 capitais brasileiras ${ }^{13}$. Considerando-se que a transmissão vertical do HIV ocorre em aproximadamente $26 \%$ das crianças expostas sem nenhuma intervenção profilática, a diferença entre este número e a taxa observada representa uma efetividade da intervenção de aproximadamente 3 em cada 4 casos. No entanto, considerando que a transmissão vertical do HIV não ultrapassa $2 \%$ quando aplicadas todas as intervenções profiláticas preconizadas, a taxa de $6,28 \%$ também mostra as oportunidades perdidas na prevenção.

A taxa geral de transmissão vertical da sífilis em Itajaí $(68,89 \%)$ foi maior do que as taxas de $47,5 \%$ a $60,6 \%$ relatadas em estudo realizado no município do Rio de Janeiro entre os anos de 1999 e 2003, em único trabalho encontrado que relata a medição direta da taxa ${ }^{28}$. Já a incidência de sífilis congênita em Itajaí $(0,3 \%)$ foi maior que aquelas descritas no ano de 2007 para o Brasil (0,18\%), Região Sudeste $(0,18 \%)$, Região Nordeste $(0,21 \%)$ e Região Norte $(0,23 \%)$. Por outro lado, a incidência em Itajaí foi três vezes maior que da Região Sul $(0,1 \%)$ e seis vezes maior que a média do Estado de Santa Catarina (0,05\%).

Embora não fosse registrado nenhum caso da transmissão vertical da hepatite $B$, vale a pena lembrar que este resultado é baseado em somente cinco recém-nascidos devidamente acompanhados após o parto, a maioria deles no ano de 2007, quando foi implementado o programa municipal de hepatites virais. Chama atenção o fato de que quase $90 \%$ das crianças nascidas de mães com hepatite $B$ não tenham recebido acompanhamento após o parto e que o teste para antígeno "e" da hepatite B nunca tenha sido feito em gestantes com os testes HBsAg e/ou anti-HBC IgM positivos. Na ausência dos testes de maior complexidade para quantificar a replicação viral, tais como o PCR, o imunoensaio para antígeno "e" é o melhor indicador de risco da transmissão vertical, tanto em gestantes recém-infectadas quanto em portadoras crônicas da hepatite B com recrudescência da replicação viral. Além disso, tampouco foi aplicado o teste de anticorpo para este antígeno, importante para avaliar a competência do sistema imunológico na presença da replicação viral, embora fosse preconizado para gestantes com resultado positivo para HBsAg e/ou anti-HBC IgM.

Os poucos estudos brasileiros sobre hepatite $\mathrm{B}$ em gestantes mostraram grande variação geográfica em percentagem do teste HBsAg positivo, de 0,22\% em São Paulo no ano de $2005^{29}$ e $0,8 \%$ no Norte do Paraná no período de 1996 a 1998, a 1\% durante a gravidez e 1,2\% no parto na área metropolitana deVitória-ES ${ }^{30}$. Somente a solicitação de rotina dos testes para hepatite $B$ durante gravidez e no parto, assim como o devido acompanhamento dos casos positivos após parto, podem elucidar melhor a transmissão vertical da hepatite B no país.

Baixa cobertura dos testes para sífilis na gravidez no começo da década de $2000^{22} \mathrm{e}$ nos meados da década ${ }^{23}$ continuam sendo 
relatados, como, por exemplo, em Feira de Santana - BA ${ }^{24}$. O maior estudo deste tipo de abrangência nacional foi baseado em 150 maternidades do SUS em 2006, e mostrou que 2,6\% das mulheres não fizeram nenhum teste para sífilis no pré-natal, sendo que $10,5 \%$ só realizaram o teste na hora de parto $^{32}$. Uma comparação com cobertura dos testes para HIV, sífilis e hepatite B maior que 95\% em gestantes na área metropolitana de Londres, no período de 2000 a $2007^{33}$, mostra como é grande a diferença na implementação dos testes para DST em gestantes entre os países.

Várias barreiras na prevenção da transmissão vertical no Brasil foram relatadas. Por exemplo, o projeto "Nascer" revelou $30,6 \%$ das maternidades com qualidade inaceitável nas ações de prevenção, vigilância epidemiológica, e assistência médica, farmacêutica e laboratorial no Nordeste ${ }^{34}$, mas o problema pode ser de abrangência nacional, embora em graus diferenciados. Além disso, alguns obstetras têm conhecimento precário da profilaxia preconizada para inibir a transmissão vertical da hepatite $\mathrm{B}^{35}$. Acrescenta-se ainda o baixo conhecimento do risco da transmissão vertical em gestantes, principalmente para hepatite $\mathrm{B}$. Como exemplo, podemos citar um estudo realizado no Acre, onde $2,1 \%$ de gestantes e $16 \%$ dos seus filhos eram positivos para o HBsAg, mas $83 \%$ das mães não sabiam que eram portadoras do vírus ${ }^{36}$.

Os exemplos citados mostram que, apesar do consenso sobre a grande importância do conhecimento da prevalência em gestantes de doenças de transmissão vertical para a formulação de políticas de saúde materno-infantil, é preciso reforçar os procedimentos preconizados pelo Ministério da Saúde para diminuir as taxas de transmissão vertical do HIV, sífilis e hepatite B no país. O monitoramento sistemático destes indicadores mostraria a efetividade das intervenções preconizadas no nível local e apontaria aos gestores do sistema de saúde os pontos específicos a serem melhorados.

\section{Referências}

1. Freitas F, Martins-Costa SH, Ramos JGL, Magalhães JA. Rotinas em obstetrícia. 4a. ed. Porto Alegre: Artmed; 2005.

2. Ministério da Saúde. Secretaria de Projetos Especiais de Saúde. Coordenação Nacional de DST/Aids. Protocolo para a prevenção de transmissão vertical de HIV e sífilis. Brasília: Ministério da Saúde; 2006.

3. Ministério da Saúde. Secretaria de Projetos Especiais de Saúde. Coordenação Nacional de DST/Aids. Boletim epidemiológico da Aids. Brasília: Ministério da Saúde; 1999.

4. Ministério da Saúde. Secretaria de Vigilância em Saúde. Programa Nacional de DST/Aids. Sífilis congênita: diretrizes para o controle. Brasília: Ministério da Saúde; 2005.

5. Ministério da Saúde. Secretaria de Projetos Especiais em Saúde. Programa Nacional de DST/Aids. Boletim epidemiológico - Aids e DSTs 2006; 3: 1-6.

6. Ministério da Saúde. Secretaria de Vigilância em Saúde. Programa Nacional de DST e Aids. Recomendações para profilaxia da transmissão vertical do HIV e terapia antiretroviral em gestantes. 4a. ed. Brasília: Ministério da Saúde; 2006.
7. De Lorenzi S, Fiaminghi LC, Artico GR. Transmissão vertical da sífilis: prevenção, diagnóstico e tratamento. Femina 2009; 37: 83-90.

8. Ramos J, Novaes A, Matida LH, Saraceni V, Veras MASM, Pontes RJS. Control of mother-to-child transmission of infectious diseases in Brazil: progress in HIV/AIDS and failure in congenital syphilis. Cad Saúde Pública 2007; 23: S370-S378.

9. FEBRASGO. Assistência pré-natal: manual de orientação. Rio de Janeiro; 2004.

10. Paz LC et al. Vigilância epidemiológica da sífilis congênita no Brasil: definição de casos, 2004. Boletim Epidemiológico-Aids e DSTs 2005; 1 e 1-2.

11. Ramos MC. Sífilis congênita, ainda um desafio [Editorial]. DST. J Bras Doenças Sex Transm 2001; 3: 2.

12. Walker DG, Walker GJ. Prevention of Congenital Syphilis - Time for Action. Bulletin of the World Health Organization 2004; 82: 6-16.

13. Vasconcelos AR, Hamann EM. Por que o Brasil ainda registra elevados coeficientes de transmissão vertical do HIV? Uma avaliação da qualidade da assistência prestada a gestantes/parturientes infectadas pelo HIV e seus recém nascidos. Rev Bras Saúde Matern Infant Recife 2005; 5: 4-12. 
14. Amaral E et al. Implementação oportuna de intervenções para reduzir a transmissão vertical do HIV: uma experiência bem-sucedida. Rev Panam Salud Publica 2007; 21: 6-13.

15. Wachholz NIR, Mascolo NP, Correa MS. Avaliação da transmissão vertical do HIV em Porto Alegre. In: XVIII Congresso Mundial de Epidemiologia/VII Congresso Brasileiro de Epidemiologia, 2008, Porto Alegre. Anais do XVIII Congresso Mundial de Epidemiologia/VII Congresso Brasileiro de Epidemiologia, Porto Alegre: ABRASCO; 2008.

16. Kummer SC. Epidemiologia das doenças infecciosas e parasitárias. In: XVIII Congresso Mundial de Epidemiologia/VII Congresso Brasileiro de Epidemiologia, 2008, Porto Alegre. Anais do XVIII Congresso Mundial de Epidemiologia/VII Congresso Brasileiro de Epidemiologia, Porto Alegre: ABRASCO; 2008.

17. Pires FG, Shama SFMS. Prevalência de HIV em gestantes testadas no centro de testagem e aconselhamento de Novo Hamburgo. In: XVIII Congresso Mundial de Epidemiologia e VII Congresso Brasileiro de Epidemiologia, Porto Alegre; 2008. Rev Bras Epidemiol (versão eletrônica); 2008 [Acessado em 23 de novembro de 2010].

18. Meireles PFM, Audi SG. Estudo da ocorrência da transmissão vertical do HIV: a realidade de Juiz de Fora. In: XVIII Congresso Mundial de Epidemiologia/ VII Congresso Brasileiro de Epidemiologia, 2008, Porto Alegre. Anais do XVIII Congresso Mundial de Epidemiologia/VII Congresso Brasileiro de Epidemiologia, Porto Alegre: ABRASCO; 2008.

19. Nishimoto TMI, Neto JE, Rozman MA. Transmissão materno-infantil do vírus da imunodeficiência humana: avaliação de medidas de controle no município de Santos. Rev Assoc Med Bras 2005; 51: 1-8.

20. Calvet GA et al. Trends in a Cohort of HIV-infected Pregnant Women in Rio de Janeiro, 1996-2004. Rev Bras Epidemiol 2007; 10: 3-11.

21. Dal Fabbro MM, Cunha RV, Paniago AM, Lindenberg Ade S, Freitas GM, Nogueira SA. Prospective study on the prevention of vertical transmission of HIV in Campo Grande, Mato Grosso do Sul, Brazil, from 1996 to 2001. Braz J Infect Dis 2005; 9: 20-7.

22. Rodrigues CS, Guimarãe MDC, César CC. Missed opportunities for congenital syphilis and HIV perinatal transmission prevention. Rev Saúde Pública 2008; 42: 851-8.

23. Chrestani MAD, Santos IS, César JA, Winckler LS, Gonçalves TS, Neumann NA. Assistência à gestação e ao parto: resultados de dois estudos transversais em áreas pobres das regiões Norte e Nordeste do Brasil. Cad Saúde Pública 2008; 24(7): 1609-18.

24. Santos, Normeide Pedreira dos; Castro, Bernardo Galvão; Rios-Grassi, Maria Fernanda. Aplicação do protocolo do Projeto Nascer Maternidades em uma maternidade de referência em Feira de Santana, Bahia, Brasil. Rev Bras Saúde Matern Infant 2009; 9: 69-76.
25. Komka MR, Lago EG, Sífilis congênita: notificação e realidade. Sci méd 2007; 17: 205-11.

26. Ministério da Saúde. Secretaria de Vigilância em Saúde. Secretaria de Atenção à Saúde. Manual de vigilância do óbito infantil e fetal e do Comitê de Prevenção do Óbito Infantil e Fetal. 2a ed. Brasília: Ministério da Saúde, 2009.

27. StataCorp. Stata Statistical software: Release 9. College Station, TX: StataCorp LP; 2005.

28. Saraceni V, Domingues RMSM, Vellozo V, Lauria LM, Dias MAB, Ratto KMN et al. Vigilância da sífilis na gravidez. Epidemiol Serv Saúde 2007; 16: 103-11.

29. Figueiredo EN, Vianna LA, Peixe MB, Ramos VM, Succi RC. The challenge of the reference and counterreference system in the prenatal assistance to pregnant women with infectious diseases. An Acad Bras Cienc 2009; 81: 551-8.

30. Lima LHM, Viana MC. Prevalence and risk factors for HIV, syphilis, hepatitis B, hepatitis C, and HTLV-I/ II infection in low-income postpartum and pregnant women in Greater Metropolitan Vitória, Espírito Santo State, Brazil. Cad Saúde Pública 2009; 25: 668-76.

31. Santos NP, Castro BG, Rios-Grassi MF. Aplicação do protocolo do Projeto Nascer Maternidades em uma maternidade de referência em Feira de Santana, Bahia, Brasil. Rev Bras Saúde Matern Infant 2009; 9: 69-76.

32. Szwarcwald CL, Barbosa Junior A, Miranda AE, Paz LC. Resultados do estudo sentinela-parturiente, 2006: desafios para o controle da sífilis congênita no Brasil. $J$ Bras Doenças Sex Transm 2007; 19(3-4): 128-33.

33. Giraudon I, Forde J, Maguire H, Arnold J, Permalloo $\mathrm{N}$. Antenatal screening and prevalence of infection: surveillance in London, 2000-2007. Euro Surveill 2009; 14: 8-12.

34. de Macêdo VC, Bezerra AF, de Frias PG, de Andrade CL. Avaliação das ações de prevenção da transmissão vertical do HIV e sífilis em maternidades públicas de quatro municípios do Nordeste brasileiro. Cad Saude Publica 2009; 25(8): 1679-92.

35. Conceição JS, Diniz-Santos DR, Ferreira CD, Paes FN, Melo CN, Silva LR. Conhecimento dos obstetras sobre a transmissão vertical da hepatite B. Arq Gastroenterol 2009; 46: 57-61.

36. Santos FCF, Vitvitski L, Paraná R, Tavares-Neto J, Acuna K, Santos Júnior R. Prevalência de infecção pelo VHB em gestantes atendidas em uma maternidade da região Amazônica brasileira. Rev Soc Bras Clin Méd 2007; 5: 1-6.

Recebido em: 24/01/11 Versão final apresentada em: 19/06/12 Aprovado em: 11/07/12 